\title{
ACUMULACIONES HOLOCENAS Y GEOARQUEOLOGIA EN EL SUR DE LA ISLA DE EUBEA (GRECIA)
}

\author{
Jose Luis PEÑA-MONNE \\ Departamento de Geografía y Ordenación del Territorio. Universidad de Zaragoza \\ Morgan DE DAPPER y Beata Maria DE VLIEGHER \\ Department of Geography. University of Gent (Belgium)
}

\begin{abstract}
Resumen: Se analizan las acumulaciones holocenas de época histórica en el sur de la isla de Eubea. Los objetivos del trabajo se centran en la datación de los depósitos que rellenan los valles y se extienden por las llanuras litorales de este sector de Grecia oriental. Igualmente, se establece la génesis de los procesos, resultantes de la actuación humana sobre las laderas entre la epoca clásica griega y romana, en unas condiciones ambientales favorables a la degradación del medio. Los resultados se ponen en relación con otras áreas de Grecia y del Mediterráneo y se discuten las diferentes interpretaciones.
\end{abstract}

Palabras clave: Geoarqueología, Grecia, Holoceno, Younger fill.

Abstract: The Holocene accumulations of historical age in the south of Eubea Island are analyzed in this work. The aims of the article are centred in the dating of the deposits that infill the valleys and are axtended over the litoral palins of this eastern Greece sector. We establish as well the genesis of processes, resulting of the human activity developed on the taluses between Greek and Roman classical ages, in environmental conditions which favour degradation of the landscape. The results are relationed with other greek and mediterranean areas, and the different interpretations are discussed.

Key words: Geoarchaeology, Greece, Holocene, Younger fill. 


\section{ANTECEDENTES Y OBJETIVOS GENERALES}

En el año 1969 se publica el trabajo de VITA-FINZI sobre los valles mediterráneos, en el que se definen dos etapas de sedimentación (Older and Younger Fill), presentándose como un modelo evolutivo de génesis climática extensible a todo el Mediterráneo. Desde ese momento, los estudios sobre geoarqueología, centrados sobre todo en los rellenos históricos, se han multiplicado en el ámbito mediterráneo. $\mathrm{El}$ avance de estos trabajos ha pasado por varias fases interpretativas (BINTLIFF, 1992), mostrando finalmente que no puede hablarse de un modelo único, sino que la realidad es mucho más compleja. Junto a las fluctuaciones climáticas del Holoceno superior, escasamente conocidas en el Mediterráneo, hay que sopesar la importancia del hombre como agente geomorfológico y la relación precisa entre las formas de explotación del medio y la erosión capaz de generar los rellenos históricos de los fondos de valle y llanuras litorales del ámbito mediterráneo.

En el Mediterráneo oriental son numerosos los trabajos publicados sobre esta temática, centrados principalmente en el Peloponeso, donde hay que destacar las publicaciones de BINTLIFF (1976 a), POPE y VAN ANDEL (1984), VAN ANDEL et al (1986), VAN ANDEL y ZANGGER (1990) y ZAMANI et al (1991) sobre la llanura de Argos y la Argólida; el estudio de DUFAURE (1976) sobre los aluvionamientos de Olimpia; o los datos obtenidos por BINTLIFF (1976 a, 1981) en la llanura de Helos, en Laconia. Otros estudios se centran en el Atica (PAEPE, 1969), en el golfo de Ambracia (DOUKELIS y FOUACHE, 1991), en la costa de Macedonia (BINTLIFF, 1976 b; DAVIDSON $(1976,1980)$ y en la costa turca del Egeo (EISMA, 1978; BINTLIFF, 1981).

Durante la realización de las campañas de campo de 1992 y 1994 para el Proyecto Global Change GC/02/027 (The Degradation of the Environment and more specifically the Desertification, in the Past, Present and Future) se localizaron en el sur de la isla de Eubea (Grecia) varios puntos de interés geoarqueológico. Desde la Llanura de Karystos hasta el cabo Paximhadi, en el extremo meridional de la isla, existen depósitos recientes de fondo de valle, laderas y llanura litoral que presentan en su interior algunos restos arqueológicos permitiendo su datación relativa. Estas acumulaciones resultan de gran interés para la reconstrucción paleoambiental de la región en época clásica y romana, por lo que se realizaron trabajos de caracterización y datación de los mismos con vistas a correlacionarlos con las cronologías y génesis de los procesos geomorfológicos del Holoceno superior obtenidos en otros lugares de Grecia.

La coincidencia en el área de trabajo con el equipo de investigadores dirigido por el Prof. Keller, dentro del proyecto de prospección SEEP (Southern Euboea Exploration Project) nos permitió obtener datós apropiados sobre la cronología de los restos arqueológicos y la localización de los yacimientos principales. 


\section{SITUACION Y CARACTERISTICAS GENERALES}

La isla de Eubea está situada (Fig. 1) en el mar Egeo, al Oeste de las regiones continentales de Atica y Beocia, de las que queda separada por un estrecho brazo de mar. El extremo meridional de la isla, a $38^{\circ} \mathrm{N}$ y $24^{\circ} 30^{\prime}$ E está dominado por las alturas del macizo montañoso del Oros Ohi $(1.398 \mathrm{~m})$ y los relieves de las penínsulas de Paximhadi y Mandhili que cierran la bahía de Karistos. Aunque se encontraron numerosos puntos con acumulaciones holocenas, nos centraremos en los depósitos de la costa oriental de Paximhadi, donde existian trabajos previos de prospección y excavación arqueológica por parte del Southern Euboea Exploration Project, ya mencionado.

Geológicamente, el área meridional de la isla de Eubea forma parte del Macizo Cicládico (PAPAVASSILOU, 1983) y tectónicamente se enmarca en el Manto Tectónico Neohelénico (KATSIKATSOS, 1991). Está formado por rocas metamórficas de gran espesor, abarcando materiales de edades mesozoico-paleógenas que se desplazaron en el Eoceno medio. Predominan las series esquistosas, con algunas intercalaciones de mármoles.

La bahía de Karistos está rodeada, como se ha indicado, por los relieves de gran energía del Monte Ohi, en los que la nieve puede permanecer varios meses en invierno gracias a su acumulación a sotavento por los vientos dominantes del Norte. Sin embargo, las penínsulas meridionales son de escasa altura y extensión, con características ambientales puramente mediterráneas.

La península de Paximhadi forma un saliente montañoso estrecho, de unos $7 \mathrm{~km}$ de longitud y alargado de $\mathrm{N}$ a $\mathrm{S}$, cerrando por el $\mathrm{W}$ la bahía de Karistos. A pesar de la intensa profundización de los torrentes, las características litológicas no permiten resaltes estructurales de importancia, predominando las aristas de divisoria estrechas y ramificadas. Una de estas crestas recorre la península a unos $200 \mathrm{~m}$ de altura (294 m de cota máxima) dividiéndola en tres cuencas-vertiente (Fig. 1): la SW, compuesta de pequeños cursos que caen con fuerte pendiente hacia una costa muy acantilada; la vertiente $\mathrm{N}$ ocupada por la cabecera de un afluente del Rigía Potamos que recorre la llanura de Karystos, y la vertiente $\mathrm{E}$, orientada hacia la bahía de Karystos. Esta última está subdividida en tres sectores arqueados: el más septentrional está limitado al $\mathrm{S}$ por un saliente rocoso y la isla de Ag. Pelagia; el central es el más amplio y queda cerrado al S por el cabo de Mnima; y el meridional, cerrado por el tómbolo del cabo Paximhadi. 

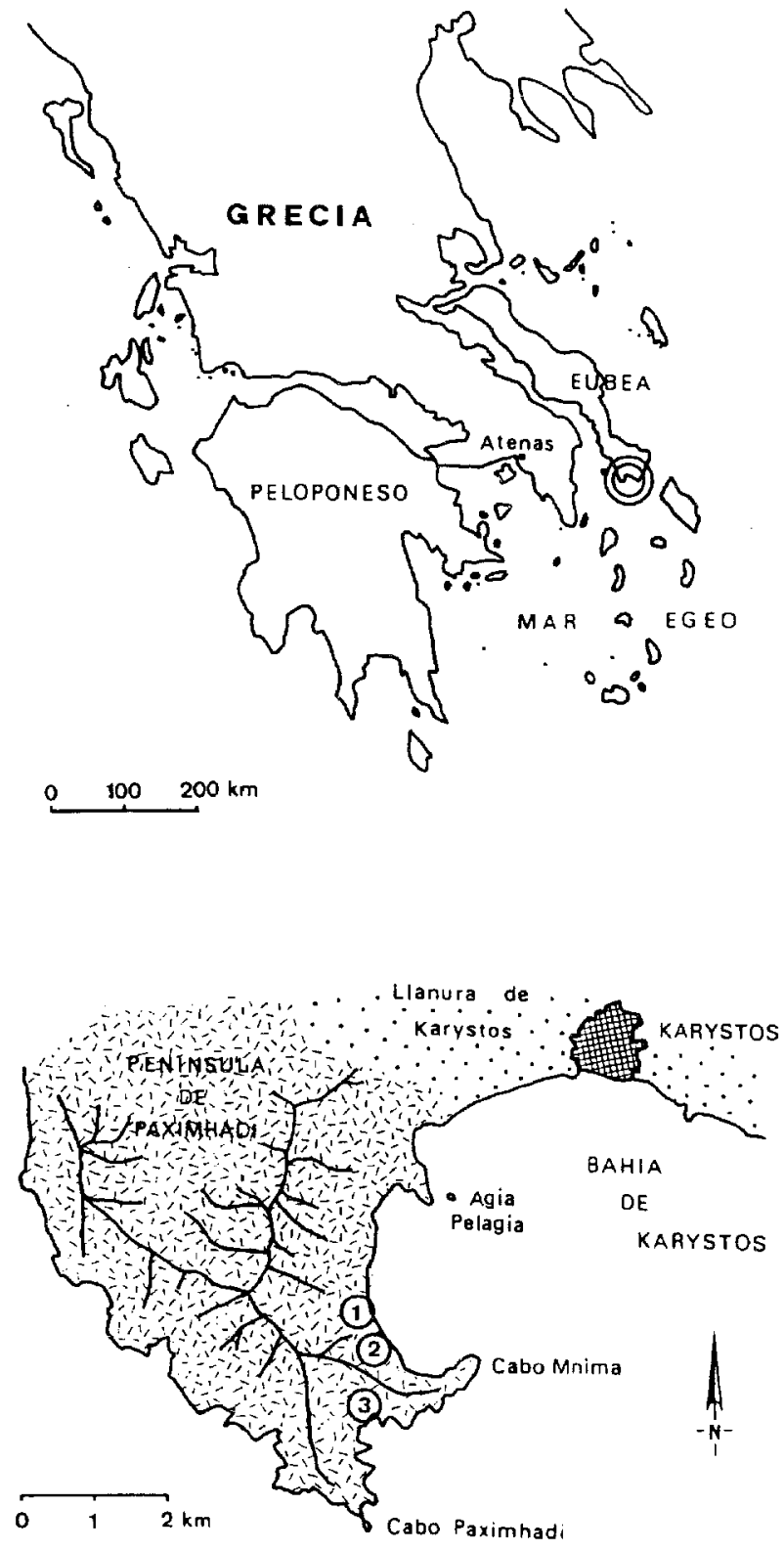

Figura 1.- Mapa de situación del área de estudio en la isla de Eubea y de las acumulaciones holocenas analizadas en la península de Paximhadi. 


\section{DESCRIPCION DE LOS RELLENOS HISTORICOS}

Se han identificado acumulaciones holocenas tanto en la llanura de Karystos como en cada uno de los sectores señalados en la vertiente oriental, aunque nos vamos a centrar en el análisis de los tres puntos de mayor interés, describiendo los rasgos arqueológicos y geomorfológicos más destacables de cada uno de ellos (Fig. 1).

\section{Palio Pithari}

En una estrecha estribación esquistosa se localiza uno de los yacimientos de época clásica griega (C-38 de KELLER y WALLACE,1988), correspondiente a una granja de los siglos V-IV B.C., cuya excavación ha mostrado una estructura simple de corral bordeado de estancias. Abandonado en el siglo IV B.C., sólo se vuelve a ocuparse en época bizantina, construyéndose sobre los restos clásicos. Al pie del yacimiento se extiende una amplia llanura acumulativa formada por la desembocadura de los dos torrentes subparalelos que rodean la estribación montañosa. En la parte alta de la misma cuenca se localiza otra granja clásica (C-54 de KELLER y WALLACE, 1988) de idéntica cronología, rodeada por un cercado de piedra.

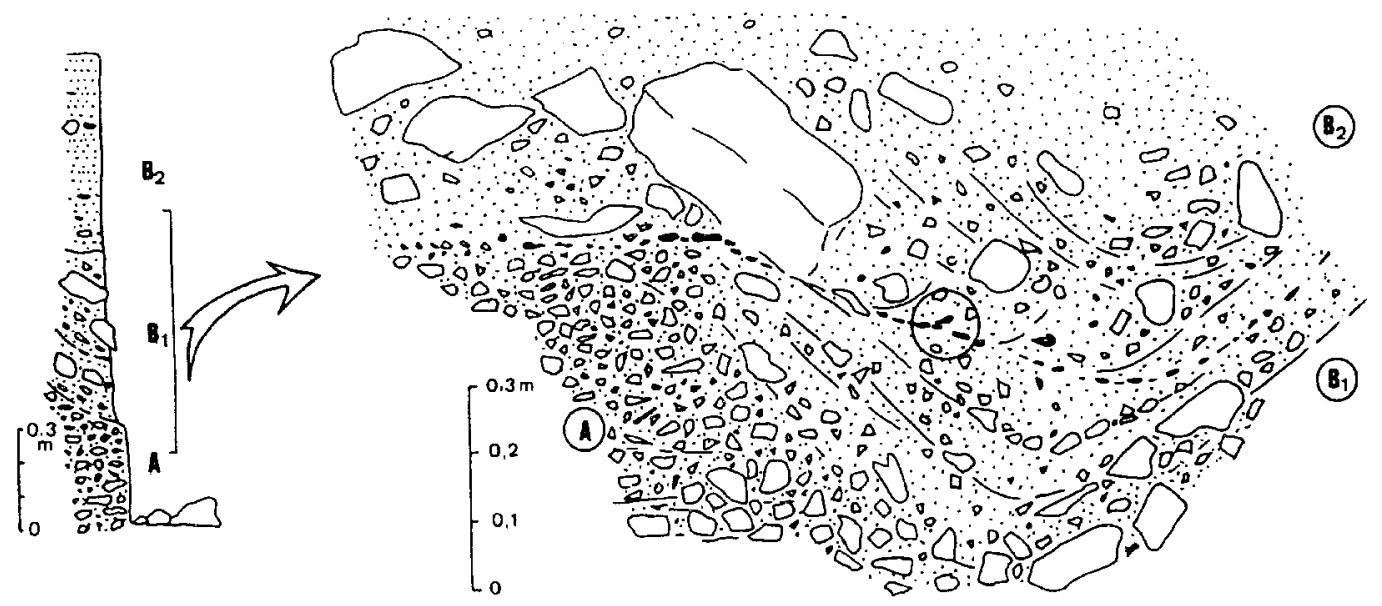

Figura 2.- Corte del relleno holoceno de Palio Pithari y detalle del nivel $B_{1}$. En negro, materia orgánica y fragmentos de carbón. El círculo indica el punto de la toma de muestra para la datación $\mathrm{C}_{14}(2080 \pm 40$ B.P.). 
La incisión existente en el depósito de la llanura aluvial nos muestra un perfil continuo, con espesor visible variable $(0,9$ a $2,10 \mathrm{~m})$. En el corte estudiado (Fig. 2) se aprecia la existencia de un nivel basal A de 1,25 $\mathrm{m}$ de espesor, fuertemente compactado y formado por cantos y bloques de esquistos escasamente ordenados, con pocas estructuras sedimentarias visibles y aspecto caótico. Por encima aparece el nivel $B_{1}$, compuesto de rellenos de canal groseros que cortan el nivel A y que localmente (como en el caso del corte de la Fig. 2) contienen nivelillos con fragmentos de carbón. También es frecuente la presencia de grandes bloques paralelipédicos que por su disposición local y dimensiones hemos relacionado con restos de muros de bancales desplazados desde las laderas. El nivel $\mathrm{B}_{2}$ forma la parte alta del relleno y se compone de capas homogéneas de material limo-arcilloso de color claro, con algunos niveles de cantos y gravillas y huellas de haber sido cultivado.

Las dataciones con C14 realizadas en las capas carbonosas del nivel $\mathrm{B}_{1}$ han dado una fecha de $2080 \pm 40$ B.P., con una edad calibrada de 58 B.C. Por lo tanto la base de este nivel es de época romana. En las cercanías del mar tanto el nivel A como los B contienen cerámicas de época clásica griega, provenientes seguramente de la erosión del yacimiento indicado, por lo que el conjunto sedimentario es de edad postclásica y la mitad superior, según la datación obtenida, es claramente de época romana.

\section{Cono de deyección de Mnima}

Está situado al sur del anterior y su dispositivo sedimentario es muy distinto a lo habitual, ya que no es un simple relleno de fondo de valle con escasa movilidad longitudinal de la carga, sino que se trata de un extenso abanico aluvial, que llegó a penetrar profundamente en el mar, depositado por un torrente de alta energía. Posteriormente la acción marina ha ido erosionando el frente del cono hasta regularizar el trazado costero, generando un acantilado detrítico rectilíneo de 1,3-1,6 $\mathrm{m}$ de altura (Fig. 3).

El ápice del cono está compuesto de bloques y gravas angulosas, disminuyendo las dimensiones del material hacia la costa, de manera que los perfiles visibles en el acantilado se componen de arenas y arcillas, con cantos dispersos, todo ello de origen continental. También se observan abundantes fragmentos de cerámicas, perfectamente clasificables como de época clásica (siglos V-IV B.C.), posiblemente procedentes del ámbito de cultivo y hábitat de un yacimiento de esa época situado en las laderas de la cabecera del torrente. Por otra parte, sobre la superficie aplanada del cono se sitúan los restos de una granja de época romana (siglos II-V A.D.) (yacimiento C-34). Por consiguiente, el abanico aluvial debió generarse en época clásica o postclásica y estaba ya modelado en época romana permitiendo el asentamiento humano sobre él. 
Así pues, el período de acumulación queda limitado entre los siglos V-IV antes de Cristo y II-V de nuestra Era.

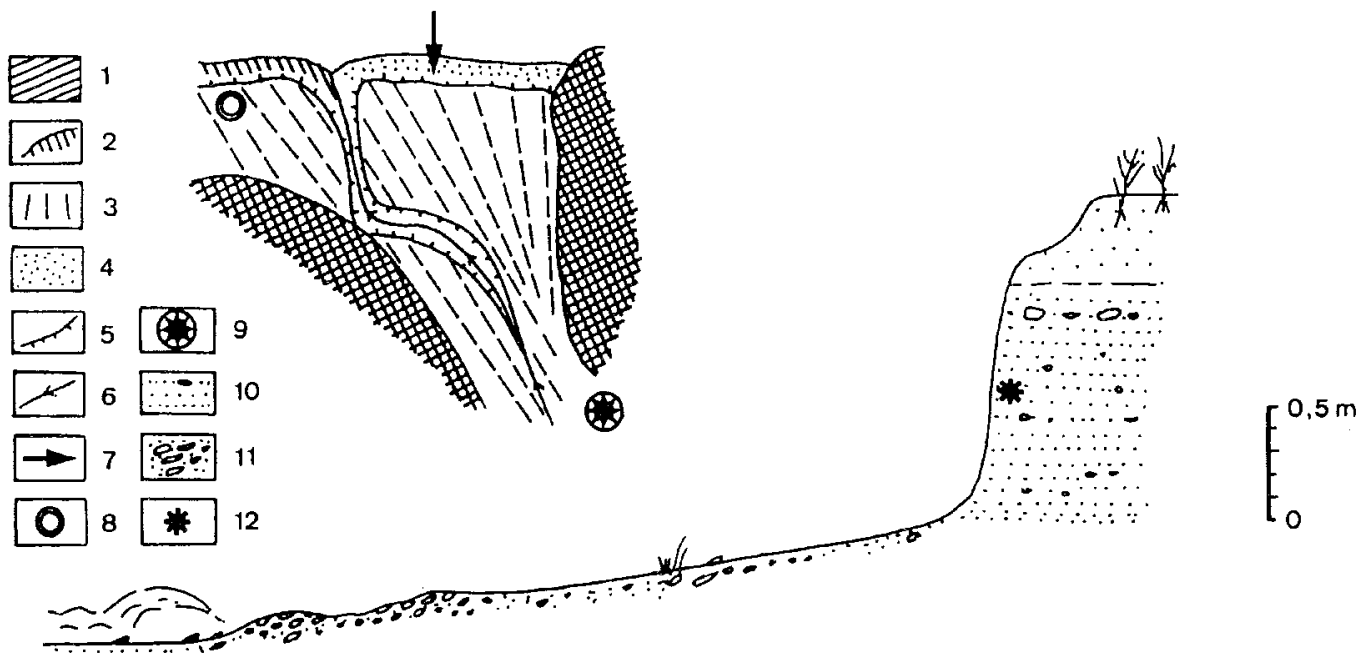

Figura 3.- Cono detrítico litoral de Mnima.

1: laderas esquistosas. 2: costa rocosa. 3: cono de deyección. 4: playa. 5: escarpes depósitos holocenos. 6: torrente. 7: situación del perfil de detalle. 8: asentamiento de época romana (s. II-V A.D.). 9: asentamiento de época clásica griega (s. V-IV B.C.). 10: depósitos del cono holoceno. 11: depósitos litorales actuales. 12: cerámica griega de época clásica.

\section{Llanura aluvial del cabo Paximhadi}

La tercera de las zonas analizadas se sitúa (Fig. 1) entre los cabos de Mnima y Paximhadi y corresponde a un relleno de fondo de valle que se prolonga hasta el litoral para enlazar con depósitos marinos. Una incisión con trazado meandriforme surca el relleno holoceno y permite observar las variaciones producidas en sentido longitudinal en las características de las acumulaciones. En conjunto se diferencian tres niveles de materiales detríticos, que se van engrosando hacia la costa, al mismo tiempo que aumenta la importancia de la incisión, pasando de $60 \mathrm{~cm}$ en la parte más interna (Fig. 4, esquema I) a 1,20 m en el sector medio-inferior (esquema II) y 1,5-1,6 m en las proximidades del litoral (esquemas III y IV). A lo largo de los perfiles se mantiene el nivel basal A formado por cantos gruesos, mientras que el nivel B es 
paulatinamente más arenoso, presentando bioturbaciones y fragmentos de carbón en el sector medio, para pasar frontalmente a formaciones litorales (IV), con una playa de cantos rodados de pumitas procedentes de Santorini; así mismo, la playa está cubierta por un nivel de arenas de una formación de dunas litorales, en cuya parte alta se aprecian signos de edafización. Finalmente, el nivel $\mathrm{C}$ corresponde a depósitos detríticos gruesos generados por procesos de descarga torrencial que fosilizan las arenas litorales; a partir de esta acumulación superior debió comenzar a generarse la incisión lineal que afecta al depósito.

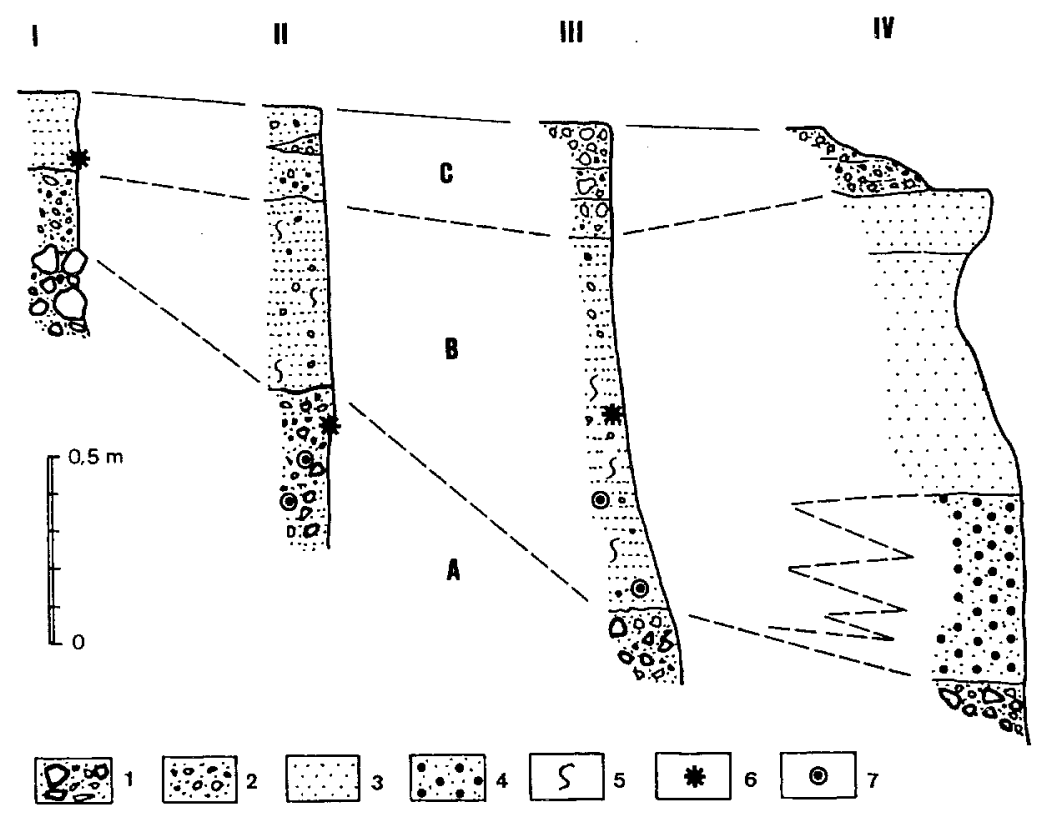

Figura 4.- Perfiles sucesivos (I-II-III) en el relleno de la llanura litoral del cabo Paximhadi hasta su enlace con los depósitos marinos (IV), mostrando tres niveles acumulativos (A, B y C). 1: gravas gruesas. 2: gravas. 3: limos y arenas. 4: playa de pumitas. 5: bioturbación. 6: fragmentos de cerámica. 7: carbones.

Las cerámicas de época clásica griega están presentes en estos perfiles, diseminadas en los tres niveles diferenciados, lo que significa que todo el depósito es de edad post-clásica, sin poder precisar más. 


\section{INTERPRETACION DE LAS ACUMULACIONES HOLOCENAS}

Existe una coincidencia bastante evidente en la cronología de las acumulaciones que ocupan los fondos de valle y litoral de la península Paximhadi, tratándose siempre de depósitos post-clásicos (post-siglos V-IV B.C.), con una clara datación de época romana (Palio Pithari). Seguramente la mayor parte de los sedimentos se acumularon en época romana y los rellenos de valle y llanuras litorales estarían ya configurados antes del siglo $\mathrm{V}$ A.D. (fecha máxima para la granja romana situada sobre el cono de Mnima).

En época clásica, en las estribaciones rocosas cercanas a los valles se instalaron algunas granjas basadas en una economía agraria, construyéndose paredes para sostener bancales estrechos y escalonados en las laderas, cuyos perfiles aún pueden deducirse en la actualidad. La morfología anterior a esta intervención humana seguramente sería una superficie regularizada por un depósito de ladera sostenido por una cobertera vegetal mediterránea. Si bien en esa época debieron comenzar ya a desencadenarse procesos erosivos, debido al cambio generado en la dinámica de las laderas y a las pendientes excesivamente fuertes en que se instalaron, sin embargo debemos considerar que en términos generales se trataba de una práctica conservacionista, la más apropiada para evitar la erosión por escorrentía superficial sobre el área de cultivo. Por otra parte, las llanuras litorales y fondos planos que ahora existen todavía no se habían configurado, de forma que estas laderas eran la principal alternativa para sostener una población agraria estable.

Los datos de la excavación de la granja clásica de Palio Pithari y otras similares de la península de Paximhadi nos muestran que su ocupación no tuvo gran continuidad y los campos debieron comenzar a ser abandonados trás un par de centurias de utilización, posiblemente por pérdida de rentabilidad de los suelos o por situaciones climáticas adversas. A partir de su abandono y del cambio en el sistema de explotación agraria, por ejemplo el paso a una sobreexplotación ganadera, la situación cambiaría, al no repararse las paredes por considerarse un espacio marginal, y al acelerarse su destrucción por la acción del ganado, que además no permitiría la recolonización vegetal natural, ayudado en muchos casos por incendios provocados. Como consecuencia de todo ello el umbral límite para el inicio de la erosión sería sobrepasado y el arroyamiento difuso y concentrado, los procesos de piping, creep, etc. se desencadenarían de forma rápida.

En los cortes de Palio Pithari (Fig. 2) se puede apreciar la presencia de bloques paralelepipédicos que en origen formarían parte de los aterrazamientos y que han sido arrastrados por la erosión hasta el fondo del valle con posterioridad a época clásica, coindiciendo en ese lugar con época romana (datación C14). Tampoco debemos olvidar la importancia del clima. El régimen de precipitaciones del Subatlántico en el 
área mediterránea debió suponer un cambio notable respecto a épocas anteriores, con lluvias de alta intensidad favorables a la generación de estos procesos de erosión. En esas circunstancias unos pocos eventos son suficientes para provocar degradaciones importantes en áreas deforestadas o donde la formación vegetal dominante es la "firghana", la única capaz de resistir la sequía estival y los incendios ligados al pastoreo (BARBERO y QUEZEL, 1983). Descargas excepcionales como las que produjeron el cono de Mnima o el nivel superior (C) del perfil del cabo Paximhadi, tendrían efectos devastadores sobre estas vertientes.

En época romana se habrían generado ya los fondos de valle y las llanuras y conos litorales de forma bastante parecida a la actual, a partir de la erosión de los bancales escalonados en las vertientes. Al formarse los rellenos de las zonas bajas, serían aprovechados posteriormente para la instalación de algunas granjas de época romana (por ejemplo el yacimiento C-34 del cono de Mnima). La propia llanura de Karystos, en cuyos cortes visibles también aparecen numerosas cerámicas clásicas y romanas, sería un buen ejemplo de estas consecuencias erosivo-sedimentarias.

En otras zonas de Grecia y del resto del Mediterráneo, se ha interpretado de diversas formas la presencia de estas acumulaciones holocenas. VAN ANDEL et al (1986) describen situaciones semejantes en la Argólida y presentan varias alternativas para explicar la relación entre expansión demográfico-cultural y procesos de morfogénesis acelerada de origen antrópico, mostrándose igualmente a favor de momentos posteriores al máximo de ocupación humana, en que comienzan a deteriorarse los muros de defensa de los bancales de cultivo, destacando la época helenística y principios de la época romana. Ello no coincide, sin embargo, con las interpretaciones presentadas en otro trabajos, que asocian directamente los ciclos de expansión y contracción humanas y de uso del territorio como causa de los procesos de erosión-sedimentación (POPE y VAN ANDEL, 1984), a pesar de que hay dificultades para correlacionar en todos los casos los eventos erosivos con los momentos históricos de superpoblación (BINTLIFF, 1992).

Un problema distinto es determinar el nivel de impacto que ha tenido la intervención del hombre en estos procesos y la importancia de la influencia del clima, todo ello en un marco de intensa actividad sísmica que puntualmente puede generar igualmente procesos destacables en esta región (BOUSQUET et al, 1983). De forma generalizada (BRUCKNER, 1986, 1990; NEBOIT, 1977, 1979; VAN ANDEL y RUNNELS, 1987; VAN ANDEL y ZANGGER, 1990, etc.) se insiste en el carácter determinante de la acción humana. Para demostrarlo, estos trabajos se apoyan en el diacronismo de los procesos a lo largo del Mediterráneo, en que las tasas de erosión en época histórica son muy superiores a las de las terrazas pleistocenas, que se suponen generadas por causas naturales, y a la ausencia de otros testimonios más claramente climáticos como terrazas fluviales o pedogénesis (BRUCKNER,1986). Sin embargo, 
las primeras explicaciones de los rellenos (VITA-FINZI, 1969) se buscaban en las fluctuaciones climáticas, basándose en el sincronismo de las acumulaciones, en la relación con las fluctuaciones climáticas del $\mathrm{N}$ de Europa y en la falta de estudios que mostraran la importancia antrópica. El modelo de VITA-FINZI (1969) era seguramente demasiado simple, tanto en la interpretación como en el número de fases existentes, sin embargo son numerosos los autores que siguen manteniendo, a pesar de considerar la acción del hombre de vital importancia, que los cambios climáticos no pueden ser desechados por completo (JORDA y VAUDOUR, 1980; BINTLIFF, 1981, 1982; NEBOIT, 1983; PEÑA et al, 1993), concediéndoles en otros casos una gran importancia, como en el NE de España para explicar el modelo de funcionamiento de los rellenos de fondos aluviales holocenos (BURILLO et al, 1985, 1986; GUTIERREZ y PENA, 1992) y como único argumento en el funcionamiento de las laderas (SANCHO et al, 1988; PEÑA y GONZALEZ, 1992).

Más dificil de explicar resulta la existencia de incisión lineal en todos los rellenos holocenos, que son los puntos de máxima actividad geomorfológica actual, correspondiendo por tanto a una etapa dinámica nueva, posterior a época romana y de la que no tenemos datos cronológicos, aunque posiblemente corresponda ya a época tardía, tal vez medieval o post-medieval. ZAMANI et al (1991) interpretan este proceso en la Argólida como debido a periodos de sequía alternando con fuertes crecidas esporádicas, es decir por causa climática. NEBOIT (1977, 1983, 1991), en los rellenos holocenos de Lucania y Sicilia, opina que esta acción lineal está producida por cambios en la pluviometría que favorecen momentos de altas aguas anuales en cauces constreñidos por sotos laterales, que impiden la instalación de crecidas sobre la llanura aluvial. JORDA y VAUDOUR (1980) opinan que puede deberse a un empeoramiento climático, una fase más fresca de época moderna o de la Pequeña Edad del Hielo, con lo que se inclinan por una causa climática.

\section{CONCLUSIONES}

En el Sur de la isla de Eubea, al igual que en otra regiones del Mediterráneo oriental, existen acumulaciones holocenas que testimonian cambios importantes en el medio en épocas históricas. Tras la instalación en época clásica (siglos V-IV antes de Cristo) de pequeñas granjas aisladas y su posterior abandono, comenzó un intenso proceso de degradación que generó importantes acumulaciones de sedimentos en valles y llanuras litorales que cambiaron notablemente la fisonomía general del lugar. La mayor parte de este proceso debió producirse ya en época romana y con anterioridad al siglo $\mathrm{V}$ después de Cristo.

A pesar de la clara relación de estos depósitos con la intervención antrópica sobre el medio, no pueden ser explicados exclusivamente por esta causa. El papel 
acelerador del hombre en los procesos históricos mediterráneos se realiza coincidiendo con cambios significativos en la dinámica climática holocena, y aunque no hay un total sincronismo, si existe un cierto ajuste local que solamente se puede explicar como consecuencia de un cambio climático general (inicio del Subatlántico) que acompañara a esta intervención humana. Esta última es ligeramente variable en el tiempo, de manera que las consecuencias de esta acción antrópica tendrían mayor o menor importancia según la altura, orientación, disposición del relieve, cobertura vegetal, etc. y se generarían de forma destacada sólo cuando el solapamiento de estos factores fuera adecuado.

Estamos de acuerdo con NEBOIT (1983) en que sin la intervención humana estos procesos no hubieran tenido lugar, o no hubieran sido tan intensos, pero no es el origen único de las acumulaciones y de la posterior incisión, sino que éstas resultan de una combinación de factores. Una prueba evidente está en el gran cambio posterior a época romana, cuando comienza el proceso de incisión, cuya causa parece estar más ligada a cambios en el clima que a los efectos que pueda producir el hombre. Es por lo tanto muy importante, trás un largo periodo de insistencia en los estudios antropogénicos, seguir investigando en las fluctuaciones climáticas holocenas y en la capacidad de éstas para provocar cambios ambientales que favorezcan la degradación del medio al intervenir sobre el mismo.

\section{BIBLIOGRAFIA}

BARBERO, M. y QUEZEL, P. (1983): La végétation de la Grèce et l'action de l'homme. Méditerranée, 48 (2), 65-71.

BINTLIFF, J.L. (1976 a): Sediments and settlement in Southern Greece. En: DAVIDSON, D.A. y SHACKLEY, M.L. (Ed.): Geoarchaeology. 267-275. Duckworth. London.

BINTLIFF, J.L. (1976 b): The plain of western Macedonian and the neolitic site of Nea Nikomedia. Proc. Prehist. Soc., 42, 241-262.

BINTLIFF, J.L. (1981): Archaeology and the holocene evolution of coastal plains in the Aegean and circum-Mediterranean. En: BROTHWELL, D. y DIMBLEBY, G. (Eds.): Environmental aspects of coast and islands. BAR Int. series, 94, 11-31. Oxford.

BINTLIFF, J.L. (1982): Climatic change, archaeology and quaternary science in the eastern Mediterranean region. En HARDING, A.F. (Eds.): Climatic change in Later Prehistory, 143161. Edinburgh Univ. Press. Edinburgh. 
BINTLIFF, J.L. (1992): Erosion in the Mediterranean lands: a reconsideration of pattern, process and methodology. En: BELL, M. y BOARDMAN, J. (Ed.): Past and Present Soil Erosion. Archaeological and Geographical Perspectives. 125-131. Oxbow Monographs 22. Oxford.

BOUSQUET, B., DUFAURE, J.J. et PECHOUX, P.Y. (1983): Temps historiques et l'évolution des paysages égéens. Méditerranée, 48(2), 3-25.

BRUCKNER, H. (1986): Man's impact on the evolution of the physical environment in the Mediterranean region in historical times. Geojournal, 13.1, 7-17.

BRUCKNER, H. (1990): Changes in the Mediterranean ecosystem during antiquity -a geomorphological approach as seen in two examples. En: BOTTEMA, S., ENTJESNIEBORG, G. y VAN ZEIST, W. (Eds.): Man's role in the shaping of the Eastern Mediterranean Landscape. 127-137. Balkema. Röterdam.

BURILLO, F., GUTIERREZ, M. y PEÑA, J.L. (1985): Las acumulaciones holocenas y su datación arqueológica en Mediana de Aragón (Zaragoza). Cuadernos de Investigación Geográfica, XI, 193-207. Logroño.

BURILLO, F., GUTIERREZ, M., PEÑA, J.L. y SANCHO, C. (1986): Geomorphological processes as indicators of climatic changes during the Holocene in the North-East Spain. En: LOPEZ VERA, F. (Eds.): Quaternary Climate in Western Mediterranean. 37-44. Univ. Autónoma de Madrid. Madrid.

DAVIDSON, D.A. (1976): Processes of tell formation and erosion. En: DAVIDSON, D.A. y SHACKLEY, M.L. (Eds.): Geoarchaeology. Duckworth. London.

DAVIDSON, D.A. (1980): Erosion in Greece during the first and second millennia B.P. En: CULLINGFORD, R.A., DAVIDSON, D.A. y LEWIN, J. (Eds.): Timescales in Geomorphology. 143-158. Wiley. London.

DOUKELLIS, P. y FOUACHE, E. (1991): L'evolution géomorphologique des deltas de l'Arachtos et du Louros. Physio-Géo, 22-23, 73-80. Meudon.

DUFAURE, J.J. (1976): La terrasse holocène d'Olympie et ses equivalents méditerranéens. Bulll. Assoc. Géogr. Franç., 85-94.

EISMA, D. (1978): Stream deposition and erosion by the Eastern Shore of the Aegean. En: BRICE, W.C. (Eds.): The Environmental History of the Near and Middle East. 67-81.

GUTIERREZ, M. y PEÑA, J.L. (1992): Evolución climática y geomorfológica del Holoceno superior (Cordillera Ibérica, Depresión del Ebro y Prepirineo). En: CEARRETA, A. y UGARTE, F.M. (Eds.): Late Quaternary in the W Pyrenaen Region. 109-124. 
JORDA, M. y VAUDOUR, J. (1980): Sols, morphogènese et actions anthropiques à l'époque historique s.l. sur les rives nord de la Méditerranée. Naturalia Monspeliensia. Coll. sur la mise en place, l'évolution et la caracterisation de la flore et de la végétation circuméditerranéenne, $\mathrm{n}^{\circ}$ hors série,173-184. Montpellier.

KATSIKATSOS, G. (1991): Geological Map of Greece 1: 50.000. Aliveri Sheet. Institute of Geology and Mineral Exploration (I.G.M.E.). Atenas.

KELLER, D.R. y WALLACE, M.B. (1987): The Canadian-Karystia Project, 1986. Echos du Monde Classique/Classical Views, XXXI (6), 225-227.

KELLER, D.R. y WALLACE, M.B. (1988): The Canadian Karystia Project: two classical farmsteads. Echos du Monde Classique/Classical Views, XXXII (7), 151-157.

NEBOIT, R. (1977): Un exemple de morphogènèse accélerée dans l'antiquité: les vallées du Basento et du Cavone en Lucanie (Italie). Méditerranée, 31, 39-50.

NEBOIT, R. (1979): Les facteurs naturels et les facteurs humains de la morphogenèse. Essai de mise au point. Ann. de Géographie, 490, 649-670.

NEBOIT, R. (1983): L'homme et l'érosion. Fac. Lettres et Sciences Humaines Univ, ClermontFerrand II, $183 \mathrm{p}$.

NEBOIT, R. (1991): Critères d'identification des facteurs d'orientation du sens de la morphogenèse fluviale en Italie du Sud et en Sicile. Physio-Géo, 22/23, 61-66. Meudon.

PAPAVASSILIOU, K. (Dir.)(1983): Geological Map of Grece 1:500.000. Institute of Geology and Mineral Exploration (I.G.M.E.). Atenas.

PAEPE, R. (1969): Geomorphic surfaces and quaternary diposits of the Adami area (south-east Attica). Thorikos, 4, 7-52.

PENAA, J.L., ECHEVERRIA, M.T., PETIT-MAIRE, N. y LAFONT, R. (1993): Cronología e interpretación de las acumulaciones holocenas de la val de las Lenas (Depresión del Ebro, Zaragoza). Geographicalia, 30, 321-332. Zaragoza.

PENAA, J.L. y GONZALEZ, J.R. (1992): Modelo evolutivo de los cambios en la dinámica geomorfológica del Baix Cinca y Segre (Depresión del Ebro) durante el Pleistoceno superior-Holoceno a partir de los datos geoarqueológicos. Cuaternario y Geomorfología, 6 , 103-110.

POPE, K.O. y VAN ANDEL, T.H. (1984): Late Quaternary alluviation and soil formation in the Southern Argolid: its history, causas and archaeological implications. Journal of Archaoelogical Science, 11, 281-306. 
SANCHO, C., GUTIERREZ, M., PEÑA, J.L. y BURILLO, F. (1988): A quantitative approach to scarp retreat starting from triangular slope facets, Central Ebro Basin, Spain. Catena Suppl. $13,139-146$.

VAN ANDEL, T.H. y ZANGGER, E. (1990): Landscape stability and destabilization in the prehistory of Greece. En: BOTTEMA, S., ENTJES-NIEBORG, G. y VAN ZEIST, W. (Eds.): Man's role in the shaping of the Eastern Mediterranean Landscape. 139-157. Balkema. Rotterdam.

VAN ANDEL, T.H., RUNNELS, C.N. y POPE, K.O. (1986): 5000 years of land use and abuse in the Southern Argolid, Greece. Hesperia, 55, 103-128.

VITA-FINZI, C. (1969): The Mediterranean Valleys. Cambridge Univ. Press. 140 p. Cambridge.

ZAMANI, A., MAROUKIAN, H. y GAKI-PAPANASTASSIOU, K. (1991): Rythmes de dépôt et de creusement pendant les temps historiques dans le cadre des sites archéologiques de la région d'Argos (Grèce). Physio-Géo, 22-23, 81-88. Meudon. 